\title{
List of Typos in Keightley's Two Articles
}

Deng Lin

Carleton College, Northfield, MN, UsA

ldeng@carleton.edu

\section{Keightley 1994}

\begin{tabular}{|c|c|c|c|}
\hline Page \# & Location & Original & Corrections \\
\hline 4 & Example $[3 \mathrm{~A}]$ & 貞 & [貞] \\
\hline 4 & {$\left[{ }_{3} \mathrm{~B}\right]$} & 貞:翌王 & [貞]: 翌王戌 \\
\hline 12 & {$[8]$} & (day 44) & (day 34 ) \\
\hline 13 & Line above $[10 \mathrm{~A}]$ & not or & or not \\
\hline 14 & {$[12 \mathrm{~A}]$} & (day ) 26, & (day 26), \\
\hline 16 & Line 12 & postive & positive \\
\hline 16 & N. 28 , Line 5 & one day day & delete extra "day" \\
\hline 24 & Line 18 & kkng & king \\
\hline 25 & Line 4 & was was & delete extra "was" \\
\hline 26 & {$[29 \mathrm{~A}],[29 \mathrm{~B}]$} & Crackmaking & Crack-making \\
\hline 26 & {$[29 \mathrm{~A}],[29 \mathrm{~B}]$} & $\mathrm{D}$ & Da? Di? \\
\hline 28 & Line 25 & bring bring & delete extra "bring" \\
\hline 30 & {$\left[35^{\mathrm{A}}\right]$} & 王寅雨 & 王寅[雨] \\
\hline 30 & {$\left[35^{\mathrm{A}}\right]$} & "Crack- & delete“ \\
\hline 32 & {$[37 \mathrm{C}]$} & kind & king \\
\hline 32 & Line 22 & auspicous & auspicious \\
\hline 32 & Bottom line & note ??? & note $41 ?$ \\
\hline 33 & {$[38 \mathrm{~A}]$} & auspciousness & auspiciousness \\
\hline 33 & N.42 & Keightley: 1978a & no “:” \\
\hline 34 & N.44 & considered & be considered \\
\hline 35 & N.46 & & $6 \mathrm{C} ?$ \\
\hline 36 & {$[41 \mathrm{~A}],[41 \mathrm{~B}]$} & jiaxu & jiawu \\
\hline 36 & {$[41 \mathrm{~A}]$} & day 11 & day 31 \\
\hline 39 & N.56 & Shaughnessy 1993: oo & Shaughnessy 1993: 62 \\
\hline 41 & Figure 1, Line 4 & {$[4 \mathrm{~A}]$} & {$\left[{ }_{4} \mathrm{~B}\right]$} \\
\hline 42 & Line 6 from bottom & qulifed & qualified \\
\hline 44 & Line 2 & 地二本 & 第二本 \\
\hline 45 & Line 17 & Zukhan & Zuhan \\
\hline 46 & Line 24 & Zhongyuang & Zhongyang \\
\hline 46 & Line 29 & yangjiuyuan & yanjiuyuan \\
\hline
\end{tabular}


(cont.)

\begin{tabular}{llll}
\hline Page \# & Location & Original & Corrections \\
\hline 47 & Line 23 & bokui & bokuji \\
47 & Line 23 & 2d & 2nd \\
47 & Line 26 & Early China 18: oo-oo & Early China 18: 41-72 \\
48 & Line 14 & Zhongyuan & Zhongyang \\
48 & Line 31 & 劉我鳥 & 劉鶚 \\
49 & Line 15 & $\ldots$ di fenqi & de fenqi \\
49 & Line 22 & & \\
\hline
\end{tabular}

Additional errors: two examples are labelled as [27] on p.25. Because of this duplication, all series numbers from that page on should be increased by one. Adjustment is also needed for examples mentioned previously at Line $4,5 \& 16$ on p.7.

\section{Keightley 1995}

\begin{tabular}{|c|c|c|c|}
\hline Page \# & Location & Original & Corrections \\
\hline 2 & N. 2 & 1994p & 1994a? \\
\hline 3 & N. 5 & 1994p & 1994a? \\
\hline 8 & Line 2 & been been & delete extra "been" \\
\hline 9 & Line 7 & knowable: & knowable. \\
\hline 9 & N.17 & Shirakawa 1962- & Shirakawa 1962-1984 \\
\hline 9 & N.18 & It may be that noted that & delete extra "that" \\
\hline 14 & N.28 & $1994 \mathrm{~T}$ & $1994 ?$ \\
\hline 15 & Line 9 & {$[24]$ and $\left[25^{B}\right]$} & {$[24]$ and $[25 \mathrm{~A}]$} \\
\hline 15 & {$[9]$} & *Make & Delete “*” \\
\hline 16 & N. 34 & period IIa & delete “a”? \\
\hline 19 & {$[15]$} & 10076 & 10067 \\
\hline 19 & N. 39 & n. 57 above & n. 57 below \\
\hline 30 & N. $5^{8}$ & n. ??? & n. 181 \\
\hline 30 & N. 6 o & $H J 116 \mathrm{~A}$ & $H J ?$ \\
\hline 33 & Line 4 from bottom & See note 143 above & below $^{1}$ \\
\hline 40 & Line 1 & 其雨's & Delete “'s" \\
\hline 41 & N. 83 & places & place \\
\hline 42 & {$\left[5^{1 \mathrm{~A}}\right]$} & {$\left[5^{1}\right] \mathrm{A}$} & {$\left[5^{1 \mathrm{~A}}\right]$} \\
\hline 45 & N. 90 & (1994T:9) & $(1994) ?$ \\
\hline
\end{tabular}

1 Herforth's explanation for this term is found in n. 69 too. 
(cont.)

Page \# Location Original Corrections

\begin{tabular}{|c|c|c|c|}
\hline 49 & N. 96 & [70] and [???] & {$[?]$} \\
\hline $5^{\circ}$ & N. 98 & "The "The & delete extra "The \\
\hline 59 & N. 121 & [???995oa]B] & HJ 995of \\
\hline 61 & Line 2 & the the more & delete extra "the" \\
\hline 62 & Line 12 & for and $[6 \circ B]$ & delete "and" \\
\hline 67 & Line 9 & ritual-sacrifce & ritual-sacrifice \\
\hline 72 & Line 1 & 則 & 斯 \\
\hline 72 & Line 11 & *tssk & *tsək \\
\hline 72 & Line 11 & *tsjgg & ${ }^{*}$ tsjəg \\
\hline 72 & Line 11 & ${ }^{*}$ tjog & *tjəg \\
\hline 72 & Line 15 & *gjøg & *gjəg \\
\hline 75 & N. $15^{2}$ & see ...... & $?$ \\
\hline 78 & Line 6 & On jisi divined (day 6) & On jisi (day 6) divined \\
\hline 79 & N. 159 & and and $T N$ & delete extra "and" \\
\hline 81,89 & {$[108 \mathrm{ABC}][12 \circ \mathrm{ABC}]$} & $\mathrm{D}$ & $\mathrm{Da}$ ? Di? \\
\hline 88 & {$[117 \mathrm{~B}]$} & *“ In & delete “*” \\
\hline 91 & Line 9 & (like $[\mathrm{iB}]$ ) & {$[1 \mathrm{C}]^{2}$} \\
\hline 95 & Line 22 & optional, & optional - \\
\hline 97 & Line 2 & childbearing,, & delete extra “,” \\
\hline 99 & Line 10 & the the twenty-four & delete extra "the" \\
\hline 99 & {$[127 \mathrm{~A}]$} & renxu (day 59) & renwu (day 19) \\
\hline 99 & {$[127 \mathrm{~B}]$} & {$[$ renxu $]$} & {$[$ renwu $]$} \\
\hline 100 & {$[128 \mathrm{~A}]$} & (day 19) & (day 59) \\
\hline 102 & Line 4 from bottom & us & is \\
\hline 111 & {$[83 \mathrm{~B}]$} & (HJ 995ob = Bingbian 9; Y535.1) & HJ 995of = Bingbian 8.1-2; $\mathrm{Y}_{536.2}$ \\
\hline 112 & N. 214 & Nivison ???? & $?$ \\
\hline 114 & Line 18 & distiguished & distinguish \\
\hline 118 & {$[138 \mathrm{~A}]$} & That evening & That day \\
\hline 119 & Line 5 & appared & appeared \\
\hline 121 & {$[14 \mathrm{OE}]$} & be be & delete extra "be" \\
\hline 124 & Line 15 & Guo Feng & Zhi Guo? \\
\hline 127 & $\mathrm{~N} .243$ & page ??? & page 210 \\
\hline 134 & Line 10 & linked to, $[142 \mathrm{~A}]$ & delete “,” \\
\hline 135 & {$[143 \mathrm{C}]$} & it will a geng-day & insert "be" after "will" \\
\hline 135 & {$[143 \mathrm{C}]$} & Yoooo & $\mathrm{Y} ?$ \\
\hline 141 & Line 15 & did referring to & did refer to \\
\hline 149 & Line 6 from bottom & the doubled used of $q i$ & the doubled use of $q i$ \\
\hline 160 & Line 3 & below & above \\
\hline
\end{tabular}

2 According to K's note, $[1 \mathrm{~B}]=[1 \mathrm{~A}]($ no qi) $(H J 339 \circ 9)$. Keightley states right after this example, on p.7, that $[1 \mathrm{C}]$ has $q i$. 
(cont.)

\begin{tabular}{|c|c|c|c|}
\hline Page \# & Location & Original & Corrections \\
\hline 165 & $\mathrm{~N} .284$ & Nivison... & Nivison? \\
\hline 166 & N.29o & of of $[155 \mathrm{IJ}]$ & delete extra "of" \\
\hline 170 & $\mathrm{~N} .293$ & See, e.g., See e.g.: & delete “See, e.g.", \\
\hline 176 & {$\left[{ }_{163} \mathrm{~B}\right]$} & $[\mathrm{L}\rfloor]$ & $(\mathrm{L}\rfloor)$ \\
\hline 185 & Line 4 & be seen divinations & insert "in" between "seen" and "divinations" \\
\hline 194 & Line 9 & putatitve & putative \\
\hline 194 & Line 15 & omisssion & omission \\
\hline 201 & Line 10 & Dagao & Da gao \\
\hline 203 & Line 12 & exhoration & exhortation \\
\hline 204 & Line 11 & desribing & describing \\
\hline 208 & Line 13 & the the back & delete extra "the" \\
\hline 228 & Line 12 & 1944 & 1994 \\
\hline 228 & Line 19 & testsugaku & tetsugaku \\
\hline 228 & Line 23 & ahong & zhong \\
\hline 230 & Line $1 \& 6$ & 凡 & 丸 \\
\hline 230 & Line 8 & & $\begin{array}{l}\text { Insert “: 殷代國家構造研究のために” after “つ } \\
\text { いて” }\end{array}$ \\
\hline 230 & Line 9 & & Insert “東” before “洋” \\
\hline 232 & Line 13 & Zhongyuan & Zhongyang \\
\hline 232 & Line 25 & $\begin{array}{l}\text { Memorial Volume of the Late } \\
\text { Professor honor of Professor }\end{array}$ & Memorial Volume in Honor of the Late Professor ${ }^{3}$ \\
\hline 233 & Line 9 & di & de \\
\hline 233 & Line 10 & & insert “甲骨探史錄” \\
\hline 233 & Line 12 & Yang, Shuda & Yang Shuda 楊樹達 \\
\hline 233 & Line 2 from bottom & & insert “動詞” after “祭祀” \\
\hline 234 & Line 2 & ০০-০o & 229-308 \\
\hline 234 & Line 4 & Hongkong & Hong Kong: Wanyou tushu. \\
\hline 234 & Last line & $19 \mathrm{pp}$. & $1994 ?$ \\
\hline \multirow[t]{8}{*}{235} & HJ 3947 & & $\mathrm{HJ} 3947 \mathrm{fb}$ \\
\hline & $\mathrm{HJ} 6016 \mathrm{fb}$ & $=[142]$ & $=[142],[161]$ \\
\hline & HJ 6482 & & $\mathrm{HJ} 6482 \mathrm{f}$ \\
\hline & HJ 6947 & & HJ $6947 f$ \\
\hline & $\mathrm{HJ} 735^{2}$ & $\mathrm{HJ} 735^{2}=[146]$ & $\mathrm{HJ} 735^{2 \mathrm{f}}=[136],[146]$ \\
\hline & HJ 11497 & & $\mathrm{HJ} 11497 \mathrm{f}$ \\
\hline & $\mathrm{HJ} 1322 \mathrm{O}$ & $\mathrm{HJ} 1322 \mathrm{Oa}$ & $\mathrm{HJ} 1322 \mathrm{Of}$ \\
\hline & HJ 19887 & & HJ 19987 \\
\hline
\end{tabular}

3 This entry, in both 1994 and 1995 papers, of Takashima (1991) "An Emphatic Verb Phrase in the Oracle-Bone Inscriptions," seems to have gotten the year wrong, should be adjusted to Takashima (1988a); page numbers seem slightly wrong too. 
The following references are missing in the List of Cited Works:

Akatsuka Kiyoshi 赤塚忠. 1977. Chūgoku kodai no shūkyō to bunka-In’ōchō no saishi 中國古代の宗教と文化 - 殷王 朝祭祀. Tokyo: Kadokawa Shoten.

Dong Zuobin 董作賓. 1931. Dagui siban kaoshi 大龜四版考釋. An Yang Fajue Baogao 安陽發掘報告 3: 423-442.

Shirakawa Shizuka 白川靜. 1962-1984. Kimbun tsûshaku 金文通釋. Hakutsuru Bijutsukanshi vol. 1-56. Kobe: Hakutsuru Bijutsukan.

Takashima, Ken-ichi. 1988. Morphology of the Negatives in Oracle-Bone Inscriptions. Computational Analysis of Asian and African Languages 30: 113-133. 